TABLE 1. Composite pressor score

\begin{tabular}{lcccc}
\hline \multicolumn{1}{c}{ Medication } & 0 points & 1 point & 2 points & 3 points \\
\hline Milrinone $(\mathrm{mcg} / \mathrm{kg} / \mathrm{min})$ & 0.0 & $<0.375$ & $0.375-5$ & $\geq 0.5$ \\
Norepinephrine & 0.0 & $<5$ & $5-10$ & $>10$ \\
$\quad(\mathrm{mcg} / \mathrm{kg} / \mathrm{min})$ & & & & \\
Epinephrine $(\mathrm{mcg} / \mathrm{kg} / \mathrm{min})$ & 0.0 & $<0.1$ & $0.1-0.4$ & $\geq 0.4$ \\
Dopamine $(\mathrm{mcg} / \mathrm{kg} / \mathrm{min})$ & 0.0 & $<4$ & $4-10$ & $>10$ \\
Dobutamine $(\mathrm{mcg} / \mathrm{kg} / \mathrm{min})$ & 0.0 & $<5$ & $5-10$ & $>10$ \\
Phenylephrine $(\mathrm{mcg} / \mathrm{kg} / \mathrm{min})$ & 0.0 & 0.2 & 0.3 & 0.4 \\
Vasopressin (U) & 0.0 & $<2$ & $2-4$ & $>4$ \\
\hline
\end{tabular}

Further studies are needed to investigate these findings. This includes repeating the analysis on other, larger patient populations; examination of other hemodynamic measures, including systemic vascular resistance, myocardial oxygen consumption, and cardiac index; the use of gene array and serum enzyme-linked immunosorbent assay techniques to identify changes in gene expression patterns during and after periods of VAD support; and examination of changes of vascular structure by using techniques such as radial artery biopsy during and after periods of CVAD support.

\section{References}

1. Jett GK. Physiology of nonpulsatile circulation: acute versus chronic support. ASAIO J. 1999;45:119-22.

2. Nishimura T, Tatsumi E, Nishinak T, et al. Diminished vasoconstrictive function caused by long-term nonpulsatile left heart bypass. ArtifOrgans. 1999;23:722-6.

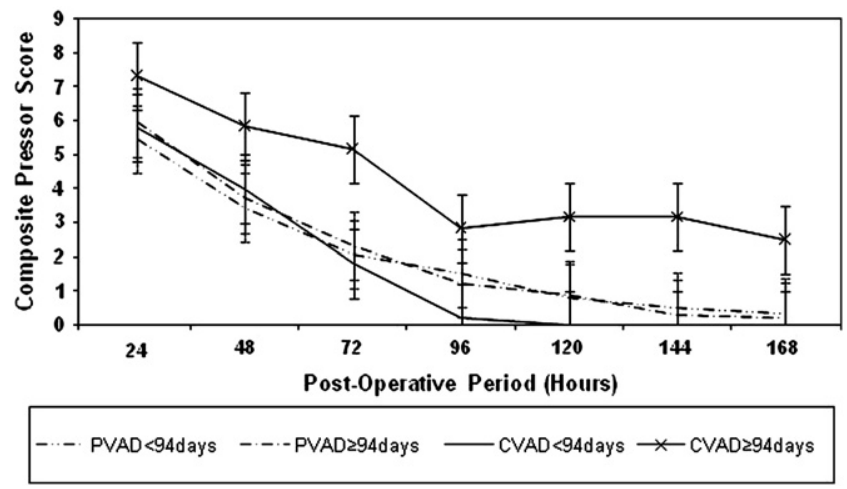

Figure 1. Composite pressor score by ventricular assist device duration and type. PVAD, Pulsatile ventricular assist device; CVAD, continuous-flow ventricular assist device.

3. Thohan V, Stetson SJ, Nagueh SF, et al. Cellular and hemodynamic responses of failing myocardium to continuous flow mechanical circulatory support using the DeBakey-Noon left ventricular assist device: a comparative analysis with pulsatile-type devices. J Heart Lung Transplant. 2005; 24:566-75.

4. Westaby S, Bertoni GB, Clelland C, et al. Circulatory support with attenuated pulse pressures alters human aortic wall morphology. J Thorac Cardiovasc Surg. 2007;133:575-6.

5. Mentzer RM Jr., Oz MC, Sladen RN, et al. Effects of perioperative nesiritide in patients with left ventricular dysfunction undergoing cardiac surgery: the NAPA Trial. J Am Coll Cardiol. 2007;49:716-26.

\title{
Superficial femoral vein as substitute for pulmonary artery reconstruction after resection for bronchovascular fistula
}

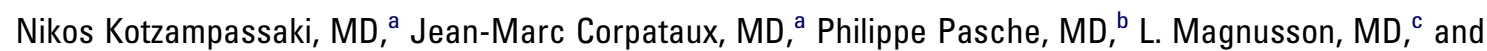

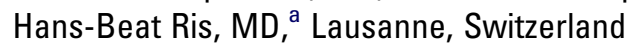

From the Divisions of Thoracic and Vascular Surgery, ${ }^{a}$ Oto-Rhino-Laryngology, ${ }^{\mathrm{b}}$ and Anesthesiology, ${ }^{\mathrm{c}}$ University Hospital of Lausanne, Switzerland.

Received for publication Aug 15, 2007; accepted for publication Oct 2, 2007.

Address for reprints: Hans-Beat Ris, MD, Centre Hospitalier Universitaire Vaudois, Service de Chirurgie Thoracique et Vasculaire, Rue du Bugnon 46, 1011 Lausanne, Switzerland.

J Thorac Cardiovasc Surg 2008;136:525-7

$0022-5223 / \$ 34.00$

Copyright $@ 2008$ by The American Association for Thoracic Surgery doi:10.1016/j.jtcvs.2007.10.071

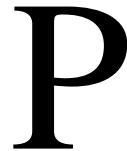

ost-lobectomy bronchovascular fistula (BVF) associated with massive hemoptysis is a rare but life-threatening complication. Surgical options include completion pneumonectomy or BVF resection with end-to-end anastomosis of the airways and reconstruction of the pulmonary artery (PA) by interposition of an appropriate substitute. We report PA resection and successful reconstruction by interposition of an autologous reversed superficial femoral vein (SFV) segment for this purpose.

\section{Clinical Summary}

A 59-year-old man with a history of coronary stenting for coronary artery disease underwent uncomplicated intrapericardial left upper lobectomy after radiochemotherapy (60 Gy) for non-small cell lung cancer. Two months later, cataclysmal hemoptysis developed in the patient, requiring cardiopulmonary reanimation, bedside rigid 


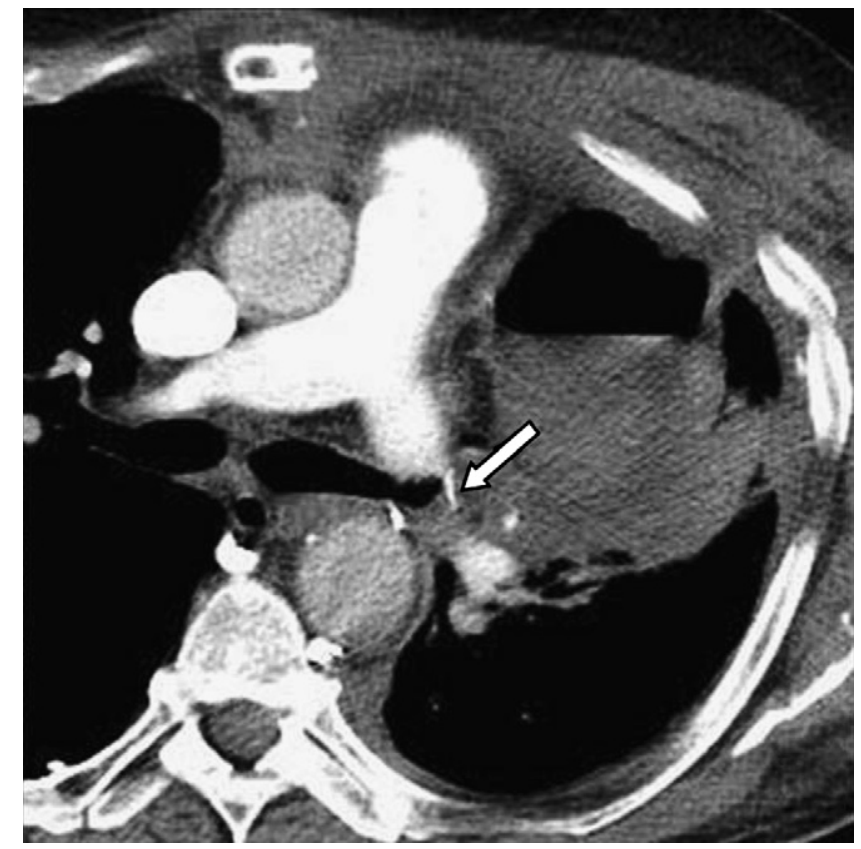

Figure 1. CT scan reveals a postlobectomy residual space containing a hematoma with an air-fluid level and air bubbles surrounding the bronchial stump which is in close contact with the pulmonary artery, suggesting a bronchovascular fistula between the bronchial stump and the pulmonary artery.

bronchoscopy, selective intubation, and stabilization in the intensive care unit. Bronchoscopy and computed tomography scan revealed a BVF between the PA and the bronchial stump (Figure 1), and surgical reintervention was considered. Posterolateral left thoracotomy was performed, and the left PA was clamped proximally and distally. Exploration confirmed the localization of the fistula between the left PA and the bronchial stump. Pneumonectomy seemed prohibitive considering the history of coronary artery disease, and segmental resection of the main stem bronchus and the PA was performed. The airway conduit was reconstructed by end-to-end anastomosis between the left main bronchus and the left lower lobe bronchus with separated suture of 5-0 polydioxanone. The PA was reconstructed by interposition of a reversed SFV segment. An interrupted suture with 5-0 Prolene was applied for both endto-end anastomoses. The reconstructed airway and PA were separated by interposition of an intrathoracically transposed serratus anterior muscle flap (Figure 2, A). Postoperative angio-computed tomography imaging at 3 and 9 months showed a patent PA reconstruction and appropriate perfusion of the residual lobe (Figure 2, B). The patient is alive and tumor-free 24 months after the operation.

\section{Discussion}

BVF is usually associated with pathologic conditions of the descending thoracic aorta, including atherosclerotic aneurysms, paraanastomotic pseudoaneurysms secondary to previous open surgical repair, and mycotic aneurysms. ${ }^{1,2}$ Massive hemoptysis caused by BVF occurring after lung surgery and involving the pulmonary arteria is a rare life-threatening event that requires urgent diagnosis and treatment. In the management of massive hemoptysis, maintenance of airway permeability is of utmost importance. The patient must be placed immediately on the involved side to prevent contralateral lung compromise. Rigid bronchoscopy should be realized without delay to achieve clot evacuation and selective intubation of the involved airway. Surgical intervention after hemodynamic and respiratory stabilization is required. ${ }^{3,4}$ Surgical options in BFV after lobectomy include completion pneumonectomy. Bronchovascular resection followed by end-to-end anastomosis of the airways and reconstruction of the PA by interposition of an appropriate substitute may be an alternative in patients unable to undergo an urgent completion pneumonectomy. Because sleeve resection with reconstruction of the PA has progressively gained acceptance as an alternative to pneumonectomy in lung cancer surgery, several PA reconstructive techniques have been proposed depending on the vascular involvement and the surgeon's attitude. ${ }^{2,3,5}$ Revascularization can be realized by patch closure or end-to-end suture when the defect is small. For extended circumferential defects in which end-to-end anastomosis is not feasible, interposition of a synthetic prosthesis (Dacron, polytetrafluoroethylene) or biologic substitute (pericardium, azygos vein) has been described. ${ }^{2,3,5}$ Biologic substitutes have a number of advantages, such as availability and biocompatibility with a reduced risk of infection and graft thrombosis, compared with synthetic substitutes. Direct contact between the
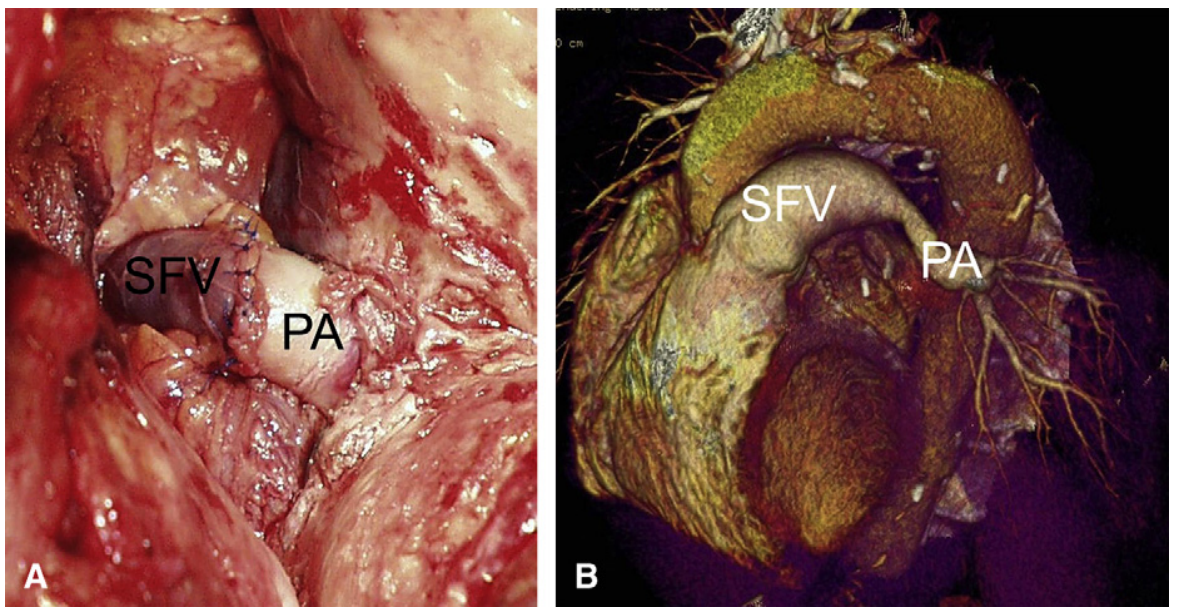

Figure 2. SFV, superficial femoral vein; PA, pulmonary artery. A, Intraoperative documentation of the pulmonary artery (PA) reconstruction by end-to-end superficial femoral vein (SFV) interposition. The distal anastomosis is shown with the lung on the right side of the picture. The proximal anastomosis is covered by a serratus anterior muscle flap transposed intrathoracically (left side of the picture). B, CT-angiography reconstructions 3 months after the operation revealing a patent reconstruction without caliber mismatch of the pulmonary artery (PA) by end-to-end superficial femoral vein (SFV) interposition. 
reconstructed airway and PA should be avoided to prevent further erosive damage and recurrence of BVA by use of muscle flap interposition. ${ }^{1}$ Because BVF with severe hemorrhage after lung resection is rare, there are no guidelines concerning the adequate surgical treatment in such an emergency situation. In the present case, completion pneumonectomy was considered inappropriate given the cardiopulmonary reanimation and history of coronary artery disease. Therefore, we performed a segmental resection of the main stem bronchus followed by end-to-end airway suture. The PA was reconstructed by the interposition of a reversed SFV segment. The 2 reconstructed structures were separated by the interposition of an intrathoracically transposed serratus anterior muscle flap. Postoperative angio-computed tomography imaging at 3 and 18 months showed a patent PA reconstruction and appropriate perfusion of the residual lobe.

\section{Conclusions}

In emergency situations, segmental defects of the PA can be rapidly and elegantly reconstructed by interposition of an autologous re- versed SFV when pericardium is not available (prior intrapericardial resections) or synthetic substitutes are avoided because of an increased risk of infections.

\section{References}

1. Piciche M, De Paulis R, Fabbri A, Chiariello L. Postoperative aortic fistulas into the airways: etiology, pathogenesis, presentation, diagnosis, and management. Ann Thorac Surg. 2003;75:1998-2006.

2. Venuta F, Ciccone AM. Reconstruction of the pulmonary artery. Semin Thorac Cardiovasc Surg. 2006;18:104-8.

3. Rendina EO, De Giacomo T, Venuta F, Ciccone AM, Coloni GF. Lung conservation techniques: Bronchial sleeve resection and reconstruction of the pulmonary artery. Semin Surg Oncol. 2000;18:165-72.

4. Pinet C, Palka W, Metras D, Thomas P, Méric B, Dumon JF. Management of an intrabronchial rupture of right main pulmonary artery. Chest. 2002; 121:988-90.

5. Galetta D, Veronesi G, Leo F, Spaggieri L. Pulmonary artery reconstruction by a custom-made heterologous pericardial conduit in the treatment of lung cancer. Lung Cancer. 2006;53:241-3.

\title{
Life-threatening impending paradoxical embolus caught "red-handed": Successful management by multidisciplinary team approach
}

\author{
Cliff K. Choong, MD, MBBS, FRCS, FRACS ${ }^{\mathrm{a}, \mathrm{b}}$ Patrick A. Calvert, ${ }^{\mathrm{a}}$ Florian Falter, ${ }^{\mathrm{a}}$ Raj Mathur, ${ }^{\mathrm{c}}$ Derek Appleton, ${ }^{\mathrm{d}}$ \\ Francis C. Wells, ${ }^{a}$ Peter M. Schofield, ${ }^{a}$ and Robin Crawford ${ }^{c}{ }^{c}$ Cambridge, United Kingdom
}

Supplemental material is available online.

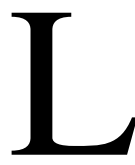

ife-threatening impending paradoxical embolus is rare. We describe a case that was caught "red-handed" and successfully treated by a multidisciplinary team approach.

\footnotetext{
From the Papworth Hospital NHS Foundation Trust, ${ }^{\text {a }}$ Cambridge, United Kingdom, ${ }^{a}$ Department of Surgery, University of Cambridge, Cambridge, ${ }^{b}$ United Kingdom, Department of Gynaecology, The Rosie Hospital NHS Trust, ${ }^{\mathrm{c}}$ Cambridge, United Kingdom, Department of Radiology, Addenbrooke's Hospital NHS Trust, ${ }^{\mathrm{d}}$ Cambridge, United Kingdom.

Received for publication Sept 3, 2007; accepted for publication Oct 19, 2007.

Address for reprints: Cliff K. Choong, MD, MBBS, FRCS, FRACS, University Lecturer, University of Cambridge, Consultant Cardiothoracic Surgeon, Papworth Hospital NHS Foundation Trust, Cambridge, CB23 8RE, United Kingdom (E-mail: cliffchoong@hotmail.com).

J Thorac Cardiovasc Surg 2008;136:527-8

$0022-5223 / \$ 34.00$
}

Copyright (C) 2008 by The American Association for Thoracic Surgery doi:10.1016/j.jtcvs.2007.10.090

\section{Clinical Summary}

A 47-year-old woman presented with dyspnea and syncope. Before the presentation, she had been bed bound for 1 week after an ankle injury. During assessment, she had signs of respiratory distress but was hemodynamically stable. Blood analysis was unremarkable except for a significantly increased D-dimer. She was treated empirically with heparin for pulmonary embolism. Computed tomographic pulmonary angiography (CTPA) of the chest revealed extensive emboli in both main pulmonary arteries with filling defects in the left and right atria (Figures E1-E3). She was transferred to a tertiary cardiothoracic center. Echocardiography revealed a 9$\mathrm{cm}$-long mobile mass within the right atrium traversing through a patent foramen ovale (PFO) into the left atrium (Figure 1, $A$ ). Whole-body computed tomography revealed an unexpected finding of a $23 \times 13 \times 12-\mathrm{cm}$ giant uterine fibroid compressing both iliac veins with extensive thrombi in the right iliac and lower limb veins (Figures E4 and E5). There was no thrombus in the left lower limb veins. She had no history of fibroid or abdominal mass. After a multidisciplinary discussion, the patient underwent surgical embolectomy of the cardiac and pulmonary emboli with closure of the PFO. A $9 \times 1.3-\mathrm{cm}$ fresh embolus was removed from the PFO (Figures $1, B, 2, A$ and $B$, and E6). An inferior vena cava filter was then deployed via the left femoral vein by an interventional radiologist, followed by an ovarian-sparing hysterectomy performed by the gynecology team (Figures E7 and E8). She had a good postoperative recovery and was discharged 8 days after surgery. She has remained well for 12 months since surgery. 